\title{
Diversity of sponge mitochondrial introns revealed by cox 1 sequences of Tetillidae
}

\author{
Amir Szitenberg ${ }^{1}$, Chagai Rot ${ }^{1,2}$, Micha llan ${ }^{1}$, Dorothée Huchon ${ }^{1,3^{*}}$
}

\begin{abstract}
Background: Animal mitochondrial introns are rare. In sponges and cnidarians they have been found in the cox 1 gene of some spirophorid and homosclerophorid sponges, as well as in the cox 1 and nad 5 genes of some Hexacorallia. Their sporadic distribution has raised a debate as to whether these mobile elements have been vertically or horizontally transmitted among their hosts. The first sponge found to possess a mitochondrial intron was a spirophorid sponge from the Tetillidae family. To better understand the mode of transmission of mitochondrial introns in sponges, we studied cox 1 intron distribution among representatives of this family.

Results: Seventeen tetillid cox 1 sequences were examined. Among these sequences only six were found to possess group I introns. Remarkably, three different forms of introns were found, named introns 714, 723 and 870 based on their different positions in the cox 1 alignment. These introns had distinct secondary structures and encoded LAGLIDADG ORFs belonging to three different lineages. Interestingly, sponges harboring the same intron form did not always form monophyletic groups, suggesting that their introns might have been transferred horizontally. To evaluate whether the introns were vertically or horizontally transmitted in sponges and cnidarians we used a host parasite approach. We tested for co-speciation between introns 723 (the introns with the highest number of sponge representatives) and their nesting cox 1 sequences. Reciprocal AU tests indicated that the intron and cox 1 tree are significantly different, while a likelihood ratio test was not significant. A global test of cophylogeny had significant results; however, when cnidarian sequences were analyzed separately the results were not significant.

Conclusions: The co-speciation analyses thus suggest that a vertical transmission of introns in the ancestor of sponges and cnidarians, followed by numerous independent losses, cannot solely explain the current distribution of metazoan group I introns. An alternative scenario that includes horizontal gene transfer events appears to be more suitable to explain the incongruence between the intron 723 and the cox 1 topologies. In addition, our results suggest that three different intron forms independently colonized the cox 1 gene of tetillids. Among sponges, the Tetillidae family seems to be experiencing an unusual number of intron insertions.
\end{abstract}

\section{Background}

Mitochondrial introns are self-splicing, selfish and mobile genetic elements [1-3]. The mobility of these introns is often facilitated by homing endonucleases (HEs) that are encoded within the introns [4,5]. Mitochondrial introns are rare in Metazoa. Both group I and group II introns have been described. Group II introns are the least frequent. They have only been found in Placozoa [6] and in an annelid worm [7]. Group I introns have been found in several unrelated Cnidaria (e.g. [8-11]), Porifera (e.g.

\footnotetext{
* Correspondence: huchond@post.tau.ac.il

'Department of Zoology, Tel-Aviv University, Tel Aviv 69978, Israel Full list of author information is available at the end of the article
}

$[12,13])$, and Placozoa (e.g., [6]). As a case in point, Tetilla sp. SP25456 (Spirophorida, [12]) and Plakinastrella onkodes (Homosclerophorida, previously identified as Plakortis angulospiculatus [13], D. Lavrov personal communication) are the only two sponges found to possess mitochondrial introns, although 22 complete mitochondrial genomes, representing a wide demosponge diversity, have already been sequenced [13-17]. A recent study of the Lebanon sponge fauna suggests that Tetilla sp. SP25456 should be synonymized with Cinachyrella levantinensis [18]. To confirm this view we sequenced a $1650 \mathrm{bp}$ fragment of the $18 \mathrm{~S}$ rRNA for both a Tetilla sp. sample from Israel and a C. levantinensis sample from
C Biomed Central

() 2010 Szitenberg et al; licensee BioMed Central Ltd. This is an Open Access article distributed under the terms of the Creative Commons Attribution License (http://creativecommons.org/licenses/by/2.0), which permits unrestricted use, distribution, and reproduction in any medium, provided the original work is properly cited. 
Lebanon (Additional file 1). The same sequence was obtained for both samples. Consequently, we use here the name C. levantinensis, rather than Tetilla sp. SP25456.

The C. levantinensis cox 1 intron was found to be 1,138 bp long [12]. Unfortunately, the cox 1 sequence of C. levantinensis is not complete. Using the complete cox 1 sequence of Amphimedon queenslandica [19] as reference, the $C$. levantinensis intron was found to be inserted after position 723. This intron encodes a putative LAGLIDADG protein. In P. onkodes two group I introns were found in the cox 1 gene but their secondary structures were not provided [13]. These introns are 388 bp and 1,118 bp in size, and are separated by 9 nucleotides (3 codons). The second intron of $P$. onkodes is inserted at the same position as the intron reported for C. levantinensis [13]. This intron and its counterpart in C. levantinensis share $81.2 \%$ nucleotide sequence identity, have a similar secondary structure, and contain LAGLIDADG ORFs. By contrast, they share only 43\% sequence identity with the first intron of $P$. onkodes, which does not contain any ORF [13].

We have previously shown that the C. levantinensis intron was more closely related to fungi introns than to any animal intron known at that time [12]. Hence we suggested that the presence of this intron in a sponge may be the result of a horizontal gene transfer event between fungi and sponges [12]. Based on a later discovery of a highly similar intron inserted at the same position in 20 scleractinian corals of the suborder Faviina [9] and in the sponge P. onkodes [13], Fukami et al. [9] and Wang \& Lavrov [13] concluded, in contrast to our hypothesis, that this intron had most likely been transmitted vertically in cnidarians and sponges, but independently lost in most lineages. However, no statistical analyses were conducted in those studies, and it is thus difficult to determine which hypothesis is better supported by the data. Interestingly, using reciprocal Shimodaira-Hasegawa (SH) tests, a Bayes factors test for incongruence, and a non-parametric version of Huelsenbeck and Bull's likelihood ratio test, Goddard et al. [10] showed that another intron, not related to the C. levantinensis intron and inserted at a different position, was horizontally transferred among actinarian cnidarians.

In order to better understand the origin and evolution of sponge mitochondrial introns we determined the cox 1 gene of 15 tetillid sponges and present statistical evidence that the $C$. levantinensis intron was both horizontally and vertically transferred in animals.

\section{Results}

Presence of mitochondrial introns and insertion sites

Fifteen new and two existing tetillid $\operatorname{cox} 1$ sequences from four genera were considered in our analysis:
Cinachyrella (9 specimens/6 species), Craniella (five specimens/4 species) Paratetilla (one specimen) and Tetilla (two specimens/two species). To avoid bias we only considered sequences that included all the insertion site positions of both the previously discovered and newly discovered tetillid introns. However, since we did not amplify complete cox 1 sequences, introns present at the beginning (the first $108-156 \mathrm{bp}$ are missing, depending on the sequence) or at the end of the gene (the last 615 - $1074 \mathrm{bp}$ ) would not be detected by our study. The complete cox 1 sequence of the sponge A. queenslandica was used as reference for numbering positions in the alignment. The use of reference sequence allowed a comparison of insertion sites between cox 1 CDSs of different lengths, as well as between partial and complete sequences. Among the 15 new sequences only five were found to possess a longer $\operatorname{cox} 1$ sequence, most probably indicating the presence of introns. Only the Cinachyrella alloclada sample was found to have an $1140 \mathrm{bp}$ long insertion after position 723. This insertion is similar in length and insertion site to those previously found for the intron of $C$. levantinensis and the second intron of P. onkodes (introns 723, Figure 1). All other insertions are shorter and inserted at different positions of the alignment. Two Cinachyrella sp. samples from Zanzibar as well as the Cinachyrella sp. 3743 sample from Australia (which probably belongs to the same species), have a 985 bp long insertion that is inserted after position 714 (introns 714, Figure 1). This insertion site is similar to that of the first intron of $P$. onkodes. Finally, the Tetilla radiata sample has a 948 bp long insertion that is inserted after position 870 (intron 870, Figure 1). Interestingly, the introns present in the cnidarians Palythoa sp. and Savalia savaglia are also inserted at this position. A BLASTX analysis [20] of the inserted sequences allowed us to identify a single LAGLIDADG ORF in each insertion.

\section{Intron secondary structures and sequence similarity}

All inserted sequences were found to fold into the canonical group I intron secondary structure, confirming that the insertions are indeed introns. However, not all introns had the same secondary structure. Three different secondary structures, corresponding to introns 714 , 723 and 870, were reconstructed from the five sequences (Figure 2). Introns 723 are represented in sponges by the introns of $C$. levantinensis, C. alloclada and the second intron of $P$. onkodes. They are characterized by an absence of P2 as well as large P6 (69-96 bp) and large P9 (113-116 bp) regions (Figure 2b, [12]). Introns 714 were identified in the three Cinachyrella sp. samples from Zanzibar and Australia. The first intron of $P$. onkodes was also identified as an intron 714 . These introns are characterized by an absence of P2 and P9 as 


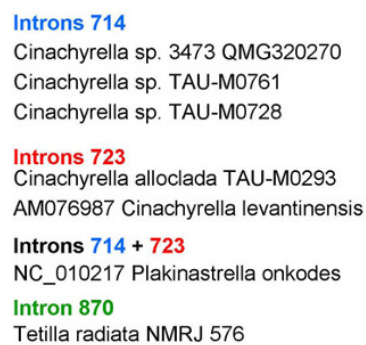

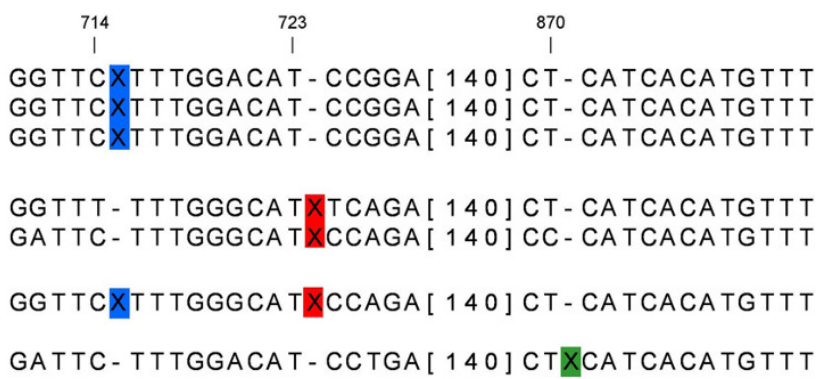

Figure 1 Intron insertion sites. Insertion sites of introns 714, 723 and 870 found in sponges are denoted by $X$. The cox 1 sequence of the sponge $A$. queenslandica was used as reference for numbering positions in the alignment.

well as the presence of a large loop ( $>10 \mathrm{bp})$ in their P1 structure (Figure 2a). It should be noted that the Cinachyrella sp. and P. onkodes intron 714 structures differ in their P5 region, which is longer in Cinachyrella sp. Finally, an intron 870 was only found in the T. radiata sequence (Figure 2c). This last intron is characterized by the presence of a P2 and a longer P5 than other sponge introns. In all introns most of the LAGLIDAGD ORF is located in the loop associated with the paired region P8. Each LAGLIDADG ORF was found to contain at least one UGA codon. This codon corresponds to a stop codon in the standard genetic code but to tryptophan in the "mold, protozoan, and coelenterate" mitochondrial code (i.e., the demosponge mitochondrial code), refuting the possibility of a nuclear origin for the introns. It is worth noting that most mitochondrial genetic codes could code for a LAGLIDADG sequence. It is thus not possible to determine the origin of the intron based on a genetic code. High sequence similarity exists among the introns inserted at the same position. The percentage of identity ranges from 85 to $99 \%$ between two sponge introns inserted at the same position, and from 43 to $53 \%$ between introns with different insertion sites.

\section{The LAGLIDADG phylogeny}

To identify the evolutionary relationships of the LAGLIDADG sequences encoded within the three intron forms, as well as their putative origin, a phylogenetic tree was reconstructed based on the LAGLIDADG protein sequences encoded within sponge introns together with similar sequences identified by a BLASTP search [21] (see Methods section). The endonuclease ORF encoded within the intron and the intron section that takes part in creating the intron's secondary structure can have different histories [1]. In this analysis, the section of the intron involved in creating its secondary structure was excluded. Consequently, the phylogenetic result allowed us to establish whether the LAGLIDADG sequences encoded within introns of similar structure and insertion sites are closely related. The resulting tree was unrooted (Figure 3). The first intron of P. onkodes was not included in this analysis since it lacked an ORF. The LAGLIDADG sequences of sponges belonged to three unrelated clades corresponding to the different insertion sites and secondary structures, which suggest that the LAGLIDADG ORFs were transmitted with their introns. Interestingly, not only the clade comprising the ORFs of introns 723 (cf. $[9,12,13]$ ) but also the clade comprising the ORFs of introns 870 included cnidarian representatives (i.e., the corals Palythoa sp. and S. savaglia [11], which had an intron inserted at the same position as $T$. radiata). The clade comprising the ORFs of introns 714, in contrast, was not found to be related to any cnidarian sequence. Another unrelated animal clade included only cnidarian sequences. The LAGLIDADG sequences of this clade originated from introns inserted at position 888. Goddard et al. [10] studied the co-phylogeny of the LAGLIDADGs encoded within introns 888 and their host.

None of the animal LAGLIDADG sequences were found to be closely related (i.e. similarity below 65\%) to any fungi or plant sequence.

\section{The cox 1 phylogeny}

To identify whether closely related introns were hosted by closely related sponges, a phylogenetic tree was reconstructed based on cox 1 sequences (Figure 4) using the maximum likelihood (ML) criterion under the $\operatorname{TrN}+\Gamma+$ I model and Bayesian analyses under the CAT $+\Gamma 4$ siteheterogeneous model [22]. The monophyly of Tetillidae was recovered with maximum support (Bayesian posterior probability: $\mathrm{PP}=1.0$; and $\mathrm{ML}$ bootstrap percentage: $\mathrm{BP}=100)$. In contrast, most of the represented tetillid genera were found to be paraphyletic. More specifically, the representative of Paratetilla was found to be closely related to Cinachyrella species. Similarly, the sequences of Tetilla leptoderma and Craniella sp. 3878 were found to be more closely related to each other than to other representatives of their respective genera. These observations indicate the need for a closer examination of the 


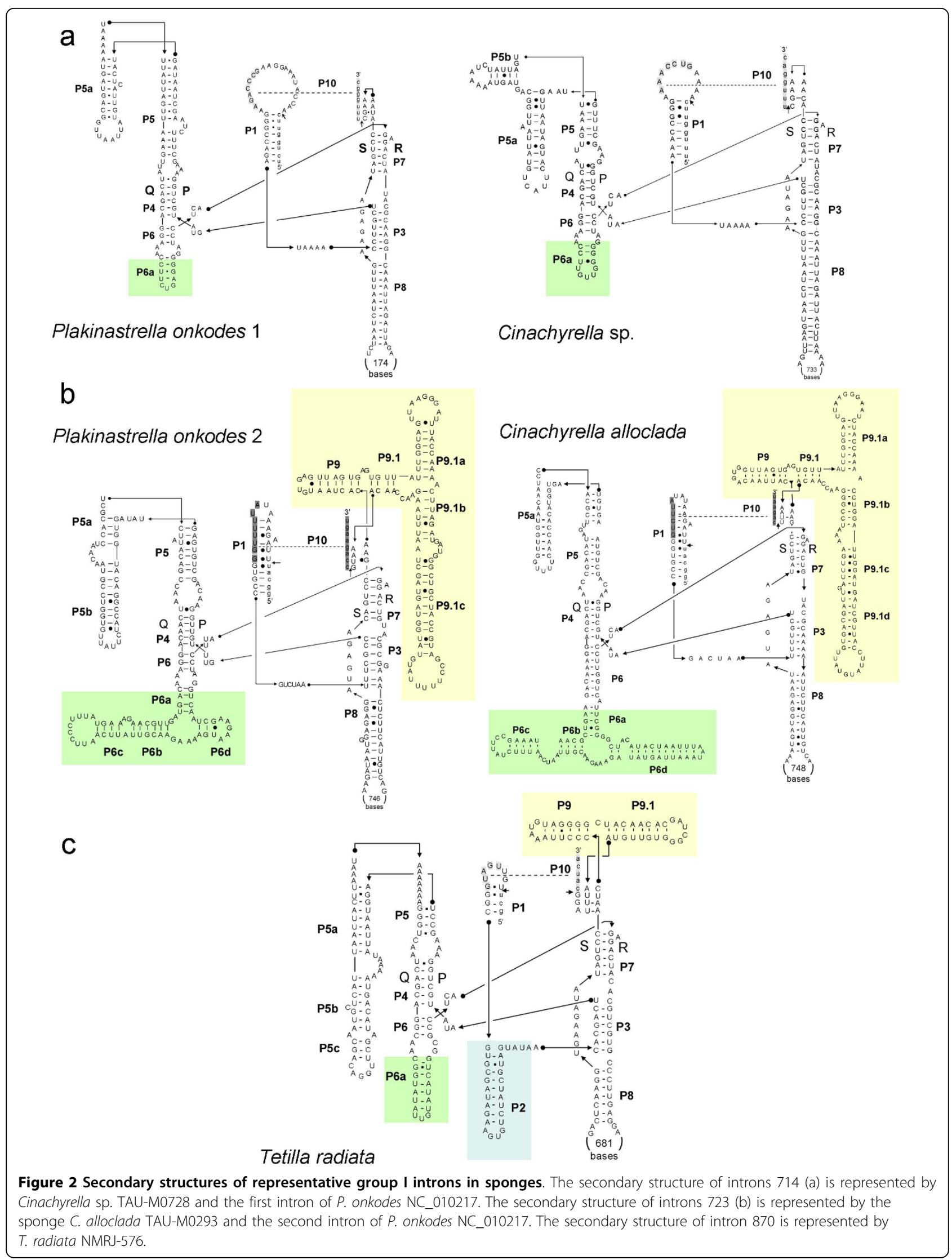




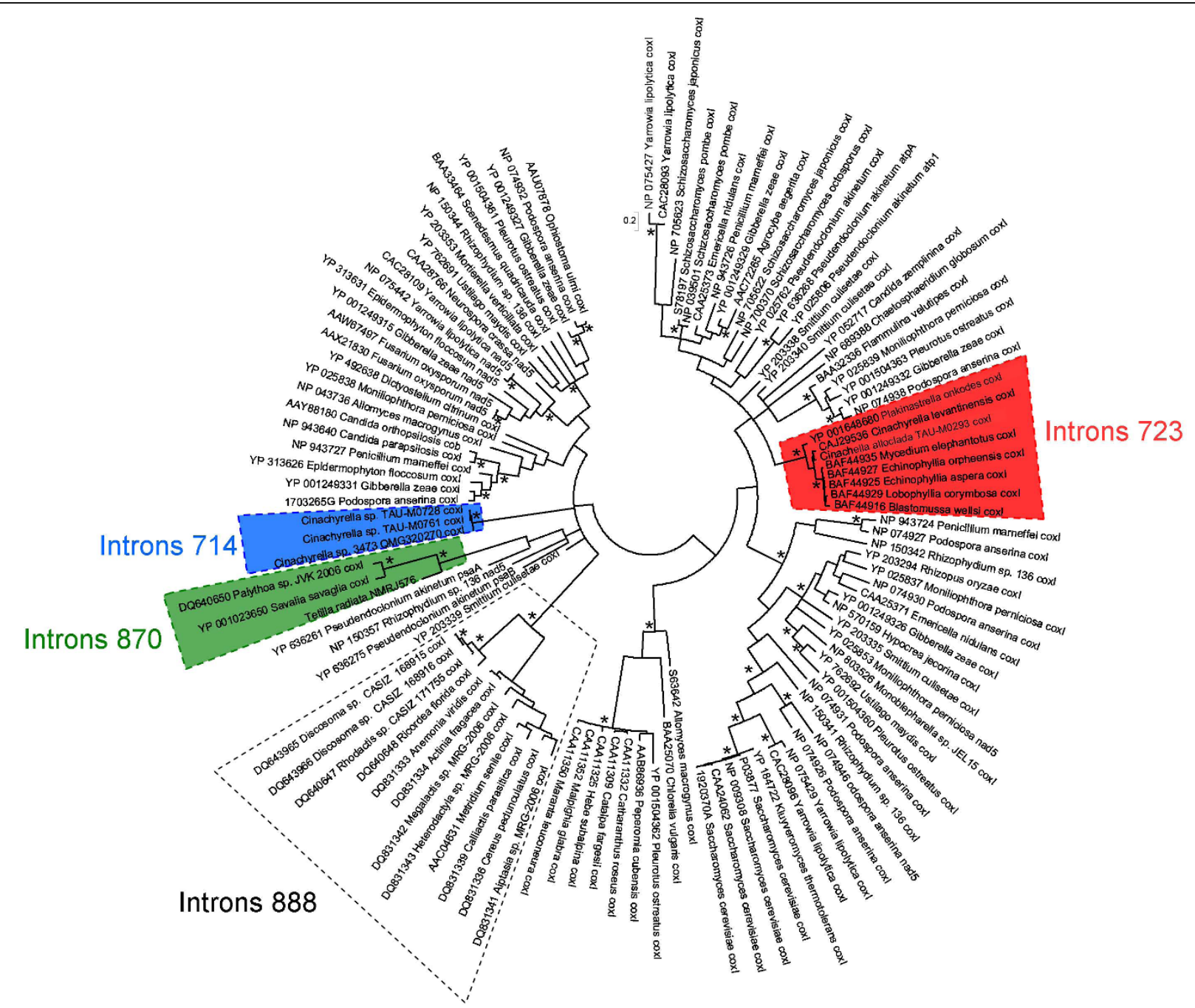

Figure 3 Phylogenetic tree reconstructed based on LAGLIDADG protein sequences. Bayesian phylogenetic tree reconstructed from LAGLIDADG protein sequences nested within group I introns of fungi, chlorophytes, streptophytes, cnidarians and sponges. Metazoan LAGLIDADG clades from introns 714, 723, 870 and 888 are delineated with a dashed line. Asterisks denote PP $>0.95$ and BP > 90. NCBI Protein accession numbers are indicated.

Tetillidae phylogeny, using additional markers and a larger taxon sampling. Surprisingly, cox 1 sequences possessing introns did not always cluster together. While the three Cinachyrella spp. specimens possessing introns 714 formed a monophyletic clade, C. alloclada and C. levantinensis, which possessed introns 723 , belong to distant lineages.

\section{Co-speciation between cox 1 sequences and their nesting LAGLIDADG sequences}

In order to examine whether the sponge introns were transmitted vertically, we checked for co-evolution between cox 1 coding sequences (CDSs) and intron sequences (the sequences included both the LAGLIDADG and the non-coding regions involved in the intron secondary structure) nesting within the $\operatorname{cox} 1$ genes. Such methods are usually used to explore cospeciation between two organisms (e.g., host - parasite relationship). In the case of cox 1 sequences and their introns it is indeed possible to consider the introns as parasites of the cox 1 genes. Since in Tetillidae we have three unrelated "parasitic" introns $(714,723,870)$, the history of each intron should be considered separately. Because only a few species were found to possess an intron 714 or an intron 870 , our co-speciation analyses were only based on introns 723. The cox 1 and intron 723 sequences of 20 corals and three sponges possessing such introns were considered in these analyses.

Following Goddard et al. [10], we first conducted tests of competing evolutionary hypotheses. More specifically, 


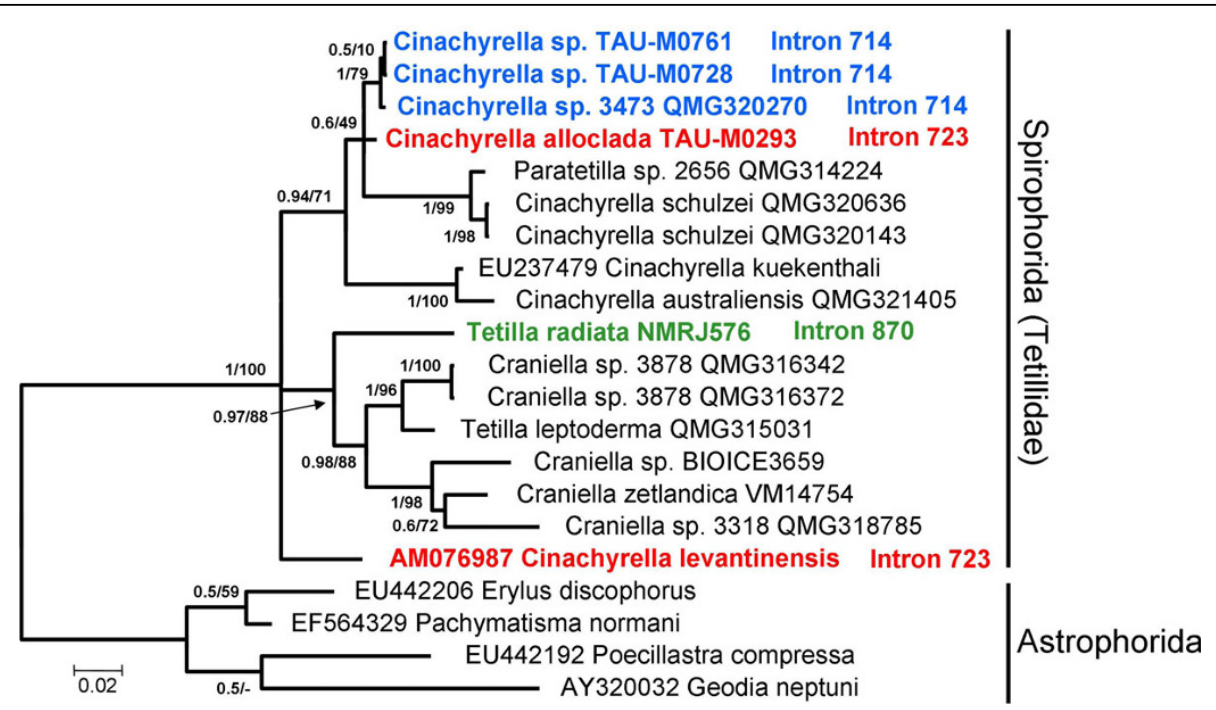

Figure 4 Phylogenetic tree reconstructed based on cox 1 coding sequence. Bayesian tree reconstructed from the cox 1 sequences of tetillids and an outgroup (astrophorid sponges). Introns are indicated next to the sequences possessing them. Posterior probabilities/ML bootstrap supports are indicated at the base of each branch. Accession numbers are indicated prior to the name of the taxon.

using reciprocal approximate unbiased (AU) tests we evaluated, for each data set separately whether the likelihood scores obtained under the intron ML topology (Figure 5a) were significantly different from the likelihood scores obtained under the cox $1 \mathrm{ML}$ topology gene (Figure 5b). Both the $\operatorname{cox} 1$ and the intron data sets reject the $\mathrm{H}_{0}$ hypothesis that the two genes could share the same topology $(p<0.001)$.

Because reciprocal AU tests are more adapted when node support is high [10] we also applied a non-parametric version of Huelsenbeck and Bull's likelihood ratio test for detecting conflicting signals [10,23]. Like the AU tests, the LRT test assumes an identical phylogeny for the $\operatorname{cox} 1$ and LAGLIDADG genes. However, in this test both data sets are not considered separately. The test computes the difference between the likelihood scores obtained when both data sets have different topologies ('two trees model') and the likelihood scores obtained when both data sets have the same topology ('one tree model'). This difference is then compared to a null distribution generated by non-parametric bootstrapping (see Methods). Unlike the AU tests, the likelihood ratio test does not reject the $\mathrm{H}_{0}$ hypothesis that the one-tree hypothesis (both genes have the same topology) is favored over the two-tree model (each gene has a different topology), although marginal significance is observed ( $p=0.072$; out of the 500 replications performed 36 were found to have a smaller loglikelihood ratio statistic than the original data set, Additional file 2).

The fact that the reciprocal AU tests reject the hypothesis of co-phylogeny and that the LRT test is marginally significant indicates the presence of at least one incongruent node between the LAGLIDADG and $\operatorname{cox} 1$ trees. These results support the hypothesis of horizontal gene transfer of introns. However they do not exclude the possibility that in some clades a vertical transmission is the most likely hypothesis. We therefore also conducted a global test of co-evolution, as well as a test on each host parasite link, using the program ParaFit [24]. In this approach, matrix permutations are used to compare $\operatorname{cox} 1$ and intron patristic distances. The analysis is thus unconstrained by the phylogenetic tree. Unlike the AU and likelihood ratio tests, the null hypothesis in ParaFit is that the host and parasite phylogenies are randomly associated, and thus that both data sets assume a different topology. Hence, a significant $p$-value indicates the existence of at least one congruent host-parasite link between the LAGLIDADG and cox 1 trees. In such a case, the tests on each host parasite link allow us to identify the co-speciating host-parasite pairs. This test revealed that the global co-speciation parameter, was significant $(p<0.001)$, albeit low (ParaFitGlobal $=0.0047)$. Only 3 out of 23 pairwise cospeciation links examined were significant, and again the link values were low $(0.0019<$ ParaFitLink $1<0.002$, $0.001<p<0.006$ ). These three significant links represented the relationships between each of the sponges sampled and their intron sequences. When these three taxa were removed from the analysis, the global test indicated no co-speciation (ParaFitGlobal $=0.00001, p>$ 0.05). The ParaFit result thus suggests that intron 723 could have been vertically transferred in tetillid and homoscleromorph sponges. However, this result is most 


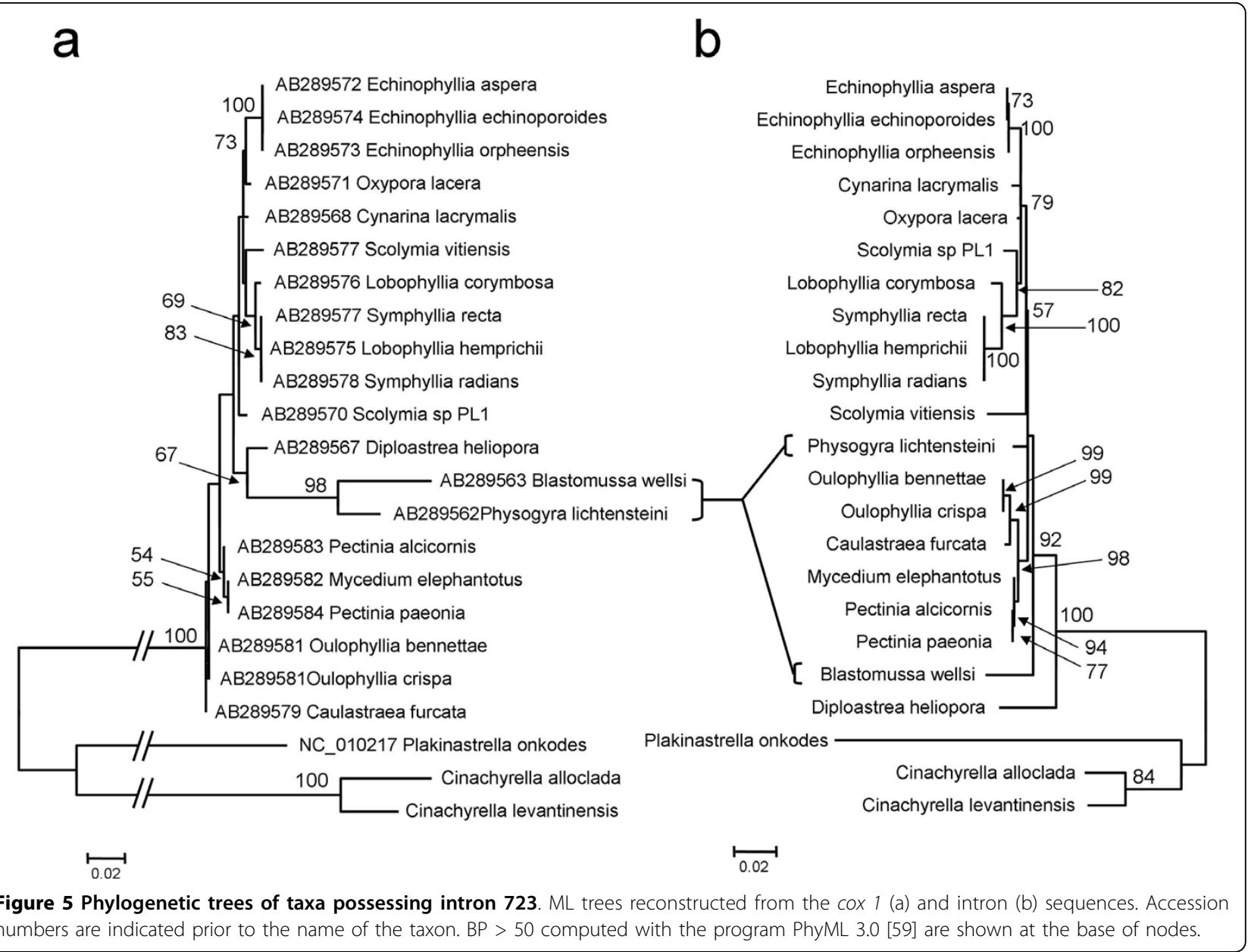

probably an artifact due to the fact that only three sponges, belonging to different distant classes, were considered in this analysis. In support of such an idea, it is worth noting that the two tetillid LAGLIDADG proteins are closer to the cnidarian sequences than to the homoscleromorph sequence $(\mathrm{PP}=0.98, \mathrm{BP}=80$, Figure 3 ). The latter result contradicts the current view that sponges are either monophyletic [25] or that homoscleromorphs are closer to cnidarians [26]. It seems thus more appropriate to explain the distribution of intron 723 in homoscleromorphs and tetillids by at least two independent transfer events. However, we cannot exclude the possibility that this intron has been vertically transmitted in tetillids.

\section{Discussion}

Mitochondrial introns are rare among metazoan organisms. Surprisingly, more than a fifth of the Tetillidae species considered in our study (4 species out of 13) were found to possess mitochondrial introns. This indicates an unusually high number of introns within this family. More surprisingly, our findings pointed to the existence of three different introns within the tetillid family. Indeed, each intron form has a different insertion site and secondary structure, and the LAGLIDADG they encode belong to unrelated clades.

There are two characteristics that might explain the presence of introns in tetillid sponges. First, sponges, similar to cnidarians, have a slower mitochondrial evolutionary rate than bilaterians $[14,27]$. This slow evolutionary rate has been suggested to facilitate the proliferation of group I introns since their splicing depends on the conservation of a rather large sequence of nucleotides $(\mathrm{n}>20)[28,29]$. Second, the transmission of genetic material is not restricted to the germline in tetillids due to their regeneration capacity [30] and budding ability [31,32]. Therefore, an intron acquired in any somatic cell has the potential to be transmitted to future generations. Interestingly, although not all members of the phylum Porifera reproduce asexually, homoscleromorphs, the second sponge lineage known to possess mitochondrial introns, also have a budding capacity [33].

Our results suggest that the family Tetillidae is a hot spot for the presence of group I introns in animals. 
However, we cannot exclude the possibility that introns might be overlooked in other sponge lineages. Indeed, two of the discovered introns are located within the reverse primer that has been recommended for the amplification of the barcoding region of the cox 1 gene in sponges [34]. As a case in point, Cárdenas et al. [35] failed to amplify the cox 1 sequence of C. alloclada using those primers, whereas we successfully amplified this species using different primers. This suggests that "standard" barcoding primers might not be adapted for the sponge and cnidarian species that share introns 714 and 870. It is therefore likely that other sponge lineages might contain mitochondrial introns, in particular those with a budding ability, for example, members the genus Tethya [36,37].

Two main scenarios can explain the distribution of these introns: one includes only vertical transmission while the second incorporates events of horizontal transfers. A scenario that includes only vertical transmission, as suggested by Wang \& Lavrov [13], would imply that the ancestor of sponges and cnidarians possessed no less than four different introns in its cox 1 sequence (Figure 3). The fact in favor of this hypothesis is that both introns 723 and 870 are shared by sponges and cnidarians. However, no species has been found to possess all four introns. Except for P. onkodes which possesses introns 714 and 723 , all other individuals with introns in their cox 1 sequence possess only a single intron. Since tetillids belong to the largest group of demosponges (the G4 clade, [38,39]), the hypothesis of a vertical transmission of the introns implies a tremendous number of independent losses in most demosponge lineages, but not in Tetillidae, whose ancestor retained three introns that were later independently lost in most tetillid species. Clearly, this scenario is improbable.

Various facts support instead the occurrence of horizontal transfer events within the mitochondrial genome of sponges. First, group I introns are known to be invasive elements that independently colonized the mitochondrial genome of numerous plants and fungi [3]. Second, the reciprocal AU tests (or SH tests [10]) on intron 723 (see Results) and intron 888 [10] support the idea that the cox 1 and intron topologies are significantly different rejecting the hypothesis of co-phylogeny. Based on the ParaFit results none of the host-parasite link is significant within Faviina corals, supporting the absence of co-phylogeny. The ParaFit results are not affected by either the node supports or the topology of the studied genes. It is possible that our ParaFit results are affected by differences in molecular-evolutionary rates between the two genes. Indeed, the $\operatorname{cox} 1$ sequences of the coral Blastomussa wellsi and Physogyra lichtensteini appear to have evolved at a faster rate than other coral species (Figure 5a), although this result might be the consequence of a misplacement of the root [9]. In contrast, the evolutionary rate of these four genera is not at odds with those of the other intron sequences (Figure $5 \mathrm{~b}$ ). Such rate differences between cox 1 and LAGLIDADG could affect the ParaFit conclusions, if they are not the result of a different history for each gene [40]. However, there are several points in favor of true phylogenetic differences between the genes. First, reciprocal AU-tests, which do not take branchlength into account, reject the hypothesis of co-phylogeny (the AU-tests are also significant in the absence of sponge sequences, data not shown). Second, Fukami et al. [9] noticed an incongruence between the cox 1 and LAGLIDADG topologies and acknowledged the possibility of horizontal transfers in scleractinian. It is therefore likely that the differences between the evolutionary rates of cox 1 and LAGLIDADG indicate a different lateral gene-transfer in Blastomussa wellsi and Physogyra lichtensteini.

Finally, although the LAGLIDADG tree suggests that the sponge and cnidarian introns have an ancient fungal origin, the specific donor of the Porifera introns is still unknown. No sequence except for cnidarian was found to be closely related to any of the sponge introns. A transfer from a cnidarian to a sponge or from a sponge to a cnidarian, as first suggested by Fukami et al. [9], appears unlikely in the case of Tetillidae since these sponges are mainly found in sediment habitats [41] and not in close proximity to corals. It is more likely that the same donor (e.g., a fungus) was at the origin of the sponge and cnidarian intron.

\section{Conclusions}

Our results support the suggestion that mitochondrial introns are both horizontally and vertically transferred in sponges and cnidarians. Given the absence of a complete phylogenetic resolution of the cox 1 and LAGLIDADG tree, the specific cases in which these introns were horizontally versus vertically transmitted still remain to be determined. Among sponges, the family Tetillidae appears as a hot spot of intron insertions, with three different intron forms present in different individuals. Interestingly, two intron forms found in sponges are closely related to cnidarian introns, suggesting that sponge and cnidarian introns might originate from a similar donor. However, the mechanisms and the donor at the origin of this transfer of genetic material remain to be discovered.

\section{Methods}

\section{Samples and molecular manipulations}

The cox 1 gene of 15 tetillid sponges was amplified. The origin of the samples is indicated in Additional file 3. 
The DNA of each sample was isolated using a modified PVP protocol [42]. The cox 1 sequences were amplified using two step nested PCRs. The conditions of PCR amplifications were: $94^{\circ} \mathrm{C}$ for $2 \mathrm{~min} ; 35$ cycles at $94^{\circ} \mathrm{C}$ for $50 \mathrm{sec}, 50^{\circ} \mathrm{C}$ for $50 \mathrm{sec}, 72^{\circ} \mathrm{C}$ for $4 \mathrm{~min}$; and a final elongation at $72^{\circ} \mathrm{C}$ for $10 \mathrm{~min}$. Different primer pairs were used depending on the species considered. The list of primer used and their sequences are indicated in Additional file 4. Amplified fragments were directly sequenced on an ABI PRISM 3100 (Applied Biosystems) genetic analyzer. The sequences were submitted to GenBank under accession numbers HM032738-HM032752.

\section{Characterization of the intron sequences}

Intron insertion-sites were determined manually, based on nucleotide and amino-acid alignments of $\cos 1$ sequences. The core structure of the $\operatorname{cox} 1$ introns was determined with Citron [43]. Peripheral hairpin structures were predicted with Mfold [44,45] using default setting. Finally, the graphic visualizations of the secondary structures were generated with RnaViz [46]. ORFs were sought for within each intron sequence. For each ORF identified, a BLASTX search [20] was conducted to determine the protein family it belonged to, if any.

\section{Phylogenetic reconstruction based on LAGLIDADG protein sequences}

Following Rot et al. [12], a LAGLIDADG dataset was constructed using BLASTP searches. Each of the LAGLIDADG sequences found in sponge introns was used as query. This dataset included mostly fungi, as well as plants, cnidarians and sponges. GenBank accession numbers are indicated in Figure 3. The protein sequence alignment was conducted with the L-INS-i algorithm under the JTT-200 substitution matrix, as implemented in Mafft version 6 [47]. Due to the high variability of the LAGLIDADG sequences, the use of Gblocks [48] or Soap [49] to remove poorly aligned region of the alignment, resulted in matrices less than 100 amino-acids (aa) long. The LAGLIDADG data set was therefore corrected manually. Sections of the alignment with more than one third of missing data were removed. Additionally, the 5' region of the LAGLIDADG ORF which takes part in the folding of the intron (i.e., the first 63 aa positions of the LAGLIDADG alignment that correspond to the largest such region) were removed as well. The final LAGLIDADG data set included 217 characters; 3 of which were constant (Additional file 5). All of the 214 variable characters were phylogenetically informative. The reconstruction of the tree was conducted with RAxML 7.0.4 [50] with 100 bootstrap repeats, under the $\mathrm{CAT}+\Gamma+\mathrm{I}$, and with Phylobayes 3.2 [51] under the CAT model. The Phylobayes analysis included two chains with 18,100 cycles
$(3,400,000$ generations) while 4,500 cycles were discarded as burnin. The maxdiff value of this run was 0.069 .

\section{Phylogenetic reconstruction based on cox 1 CDS}

The $\operatorname{cox} 1$ data set included the 15 DNA sequences obtained as well as two tetillid sequences available in GenBank. Since Astrophorida has been shown to be the sister clade of Spirophorida (e.g. [38,39]), four astrophorid sequences were used as outgroup. Accession numbers are indicated in Figure 4. A codon alignment of the $\operatorname{cox} 1$ sequences was obtained using the online version of Pal2Nal [52]. The underlying protein reference data set was aligned using MAFFT version 6 [47] with the L-INS-i algorithm. The program Gblocks [48] was then used to exclude regions that were poorly aligned. The nucleotides downstream to the intron insertion site (i.e., 18 nucleotides after each insertion) were also removed since co-conversion of cox 1 exonic sequences can occur after intron insertion [53]. The cox 1 data set used in the phylogenetic analysis included 927 characters; 687 of them were constant and 303 had missing data. Among the 240 variable sites 190 were phylogenetically informative (Additional file 6). Phylogenetic reconstruction was conducted using both the maximum likelihood (ML) and the Bayesian approaches. The ML tree was reconstructed with PAUP* 4 [54] under the $\operatorname{TrN}+\Gamma+\mathrm{I}$ model of sequence evolution and using the tree bisection reconnection (TBR) branchswapping algorithm and 100 random sequence addition starting trees. The $\operatorname{TrN}+\Gamma+\mathrm{I}$ model was found to be the best fitting ML model using Modeltest 3.7 [55]. Branch supports were estimated based on 100 bootstrap repetitions. A Bayesian tree was reconstructed with Phylobayes 3.2c [51] under the GTR CAT model of sequence evolution. The analysis included two chains with a total run length of 13,000 cycles $(490,000$ generations) while 3200 cycles were discarded as burnin. The maxdiff value of this run was 0.038 .

\section{Co-speciation between "host" (cox 1 coding sequences) and "parasite" (introns 723)}

Three different introns were found in sponges (see Results section). However, co-speciation tests, between intron sequences and their cox 1 host sequence, could only be performed for intron 723 . Other introns did not include enough representatives, except intron 888, which was already studied by Goddard et al. [10].

A total of 20 species of corals and three species of sponges possessing intron 723 were considered. The nucleotide data sets of each gene were aligned with MAFFT version 6 [47] with the L-INS-i algorithm. After manual removal of ambiguously aligned positions the $\operatorname{cox} 1$ and intron data sets were respectively 630 and 
$1078 \mathrm{bp}$ long (Additional files 7, 8. The program PAUP* 4 [54] was used to reconstruct the evolutionary relationships based on cox 1 and intron sequences, and to obtain the corresponding matrices of patristic distances. The two ML phylogenetic trees were obtained using the TBR branch-swapping algorithm under the best model of sequence evolution identified by Modeltest 3.7 [55].

Three co-speciation tests were conducted. Following Goddard et al. [10] the first approach was to compare the ML topology of both genes under each data set. Reciprocal approximate unbiased tests as implemented in Consel 0.1i [56] were applied. The site-wise log-likelihoods were obtained using PAUP* 4 [54]. The second approach was to perform a non parametric version of Huelsenbeck and Bull's likelihood ratio test [23]. This test compares the log-likelihood $L_{O}$ obtained under the hypothesis that the cox 1 gene and the intron share a single tree (each marker being allowed to evolve under different model and branch-length parameters), with the $\log$-likelihood $L_{1}$ obtained under the hypothesis that each data set evolved independently, resulting in different trees and sets of parameters. Computations were performed with PAUP* under the GTR $+\Gamma+$ I model for both gene. The log-likelihood ratio statistic $(d)$ is defined as:

$$
d=2\left(\ln L_{0}-\ln L_{1}\right)
$$

The null distribution of $d$ was obtained using nonparametric bootstrap. Five hundred bootstrap files were generated separately for the $\operatorname{cox} 1$ and the intron data sets using Mesquite 2.72 [57]. Each cox 1 bootstrap file was concatenated with one LAGLIDADG bootstrap file. The $\operatorname{cox} 1$ and intron sequences were then randomized within each concatenated file using a Perl script designed for this purpose. Three ML trees were constructed for each data set. The first was based on the first 630 positions of the concatenated and randomized matrix, the second on the last 1078 positions and the third on the total matrix length (1708 bp). The tree reconstructions were performed with PAUP* using rounds of heuristic searches starting with a neighborjoining (NJ) tree and using tree bisection-reconnection (TBR) branch-swapping. The initial model parameter values were those estimated by Modeltest. After a first round of heuristic search the parameters were estimated on the resulting tree and then used for the subsequent round of heuristic search. The process was repeated until all parameters converged. Ln $L_{0}$ was computed by assuming that the first $630 \mathrm{bp}$ and the last $1078 \mathrm{bp}$ shared the ML tree obtained using the whole data set (albeit each partition was allowed to evolve under different model and branch-length parameters). Ln $L_{1}$ was computed by assuming that the first $630 \mathrm{bp}$ and the last
$1078 \mathrm{bp}$ had different trees and different models of evolution. The third approach applied the program ParaFit [24] to perform a global test of co-speciation (ParaFitGlobal) as well as local tests for each cox 1 - intron link. Following the recommendation of the ParaFit manual, the only parameter considered was ParaFitLink1. This parameter is indeed more adapted when the global test is significant but the local tests show a mixed trend. The principle coordinates (PCOs) of the patristic matrices were calculated with DistPCoA [58] using the Lingoes correction.

\section{Additional material}

Additional file 1: 18S rRNA sequences of Tetilla sp. from Israel and Cinachyrella levantinensis from Lebanon. DNA sequences alignment in nexus format.

Additional file 2: The null distribution of the log-likelihood ratio statistic (d). The null distribution of the log-likelihood ratio statistic (d) generated by the LRT analysis of co-evolution.

Additional file 3: Origin of the tetillid samples used in this study A table describing the voucher number, GenBank accession number, geographical origin and contributor of each sample sequenced in this study.

Additional file 4: PCR primers used in this study. The sequences of PCR primers and their utilization in this study, specified for each specimen, are described in this file

Additional file 5: LAGLIDADG protein alignment. Protein sequence alignment (in Nexus format) used to reconstruct the phylogenetic tree present in Figure 3.

Additional file 6: Cox 1 DNA alignment. DNA sequence alignment (in Nexus format) used to reconstruct the phylogenetic tree present in Figure 4

Additional file 7: DNA alignment of the cox 1 sequences from taxa possessing introns 723. Cox 1 sequence alignment in Nexus format used in the co-evolution analyses.

Additional file 8: DNA alignment of the sponge and cnidarian introns 723. Intron sequence alignment in Nexus format used in co-evolution analyses.

\section{Acknowledgements}

We would like to thank the following people: Paco Cárdenas, Hans Tore Rapp, John Hooper, Eduardo Hajdu and Jean Vacelet for providing the tissue samples of tetillid sponges; Naomi Paz for editing the text and Tamar Feldstein for her advice at the bench; Nicole Boury-Esnault, Emmanuel Douzery and Nicolas Galtier for comments; and the Israeli Ministry of Science Culture \& Sport for supporting the National Collections of Natural History at Tel-Aviv University. This research was supported by the Israel Science Foundation (grant No.600/06 to D.H). D.H. is supported by the National Evolutionary Synthesis Center (NESCent), NSF \#EF-0905606.

\section{Author details}

'Department of Zoology, Tel-Aviv University, Tel Aviv 69978, Israel. 2Department of Molecular Genetics, Weizmann Institute of Science, Rehovot 76100, Israel. ${ }^{3}$ National Evolutionary Synthesis Center, 2024 W. Main St., Suite A200, Durham, NC 27705, USA.

\section{Authors' contributions}

DH conceived the study and supervised the sequencing and analyses. Ml collected and identified the biological specimens. AS and CR carried out the sequencing. AS conducted the phylogenetic analysis. AS and DH drafted the 
manuscript. All other authors assisted in writing the manuscript. All authors read and approved the final manuscript.

Received: 4 May 2010 Accepted: 20 September 2010

Published: 20 September 2010

\section{References}

1. Burt A, Koufopanou V: Homing endonuclease genes: the rise and fall and rise again of a selfish element. Current Opinion in Genetics \& Development 2004, 14(6):609-615.

2. Chevalier BS, Stoddard BL: Homing endonucleases: structural and functional insight into the catalysts of intron/intein mobility. Nucleic Acids Research 2001, 29(18):3757-3774.

3. Haugen $P$, Simon DM, Bhattacharya D: The natural history of group I introns. Trends in Genetics 2005, 21(2):111-119.

4. Belfort M: Two for the price of one: a bifunctional intron-encoded DNA endonuclease-RNA maturase. Genes \& Development 2003, 17(23):2860-2863.

5. Lang BF, Laforest MJ, Burger G: Mitochondrial introns: a critical view. Trends in Genetics 2007, 23(3):119-125

6. Dellaporta SL, Xu A, Sagasser S, Jakob W, Moreno MA, Buss LW, Schierwater B: Mitochondrial genome of Trichoplax adhaerens supports Placozoa as the basal lower metazoan phylum. Proceedings of the National Academy of Sciences of the USA 2006, 103(23):8751-8756.

7. Valles $Y$, Halanych KM, Boore JL: Group II introns break new boundaries: presence in a Bilaterian's genome. PLoS One 2008, 3(1)

8. Beagley $C T$, Okada NA, Wolstenholme DR: Two mitochondrial group I introns in a metazoan, the sea anemone Metridium senile: One intron contains genes for subunits 1 and 3 of NADH dehydrogenase. Proceedings of the National Academy of Sciences of the USA 1996, 93(11):5619-5623.

9. Fukami H, Chen CA, Chiou CY, Knowlton N: Novel group I introns encoding a putative homing endonuclease in the mitochondrial cox 1 gene of scleractinian corals. Journal of Molecular Evolution 2007, 64(5):591-600.

10. Goddard MR, Leigh J, Roger AJ, Pemberton AJ: Invasion and persistence of a selfish gene in the Cnidaria. PLoS One 2006, 1(1)

11. Medina M, Collins AG, Takaoka TL, Kuehl JV, Boore JL: Naked corals: Skeleton loss in Scleractinia. Proceedings of the National Academy of Sciences of the USA 2006, 103(24):9096-9100.

12. Rot C, Goldfarb I, llan M, Huchon D: Putative cross-kingdom horizontal gene transfer in sponge (Porifera) mitochondria. BMC Evolutionary Biology 2006,6 .

13. Wang $X$, Lavrov DV: Seventeen new complete mtDNA sequences reveal extensive mitochondrial genome evolution within the Demospongiae. PLoS One 2008, 3(7):e2723.

14. Lavrov DV, Forget L, Kelly M, Lang BF: Mitochondrial genomes of two demosponges provide insights into an early stage of animal evolution. Molecular Biology and Evolution 2005, 22(5):1231-1239.

15. Lavrov DV, Lang BF: Transfer RNA gene recruitment in mitochondrial DNA. Trends in Genetics 2005, 21(3):129-133.

16. Erpenbeck D, Voigt $O$, Wörheide G, Lavrov DV: The mitochondrial genomes of sponges provide evidence for multiple invasions by repetitive hairpin-forming elements (RHE). BMC Genomics 2009, 10.

17. Belinky F, Rot C, llan M, Huchon D: The complete mitochondrial genome of the demosponge Negombata magnifica (Poecilosclerida). Molecular Phylogenetics and Evolution 2008, 47(3):1238-1243.

18. Vacelet J, Bitar G, Carteron S, Zibrowius H, Perez T: Five new sponge species (Porifera: Demospongiae) of subtropical or tropical affinities from the coast of Lebanon (eastern Mediterranean). Journal of the Marine Biological Association of the United Kingdom 2007, 87:1539-1552.

19. Erpenbeck D, Voigt O, Adamski M, Adamska M, Hooper JNA, Wörheide G, Degnan BM: Mitochondrial diversity of early-branching metazoa is revealed by the complete $\mathrm{mt}$ genome of a haplosclerid demosponge. Molecular Biology and Evolution 2007, 24(1):19-22.

20. Gish W, States DJ: Identification of protein coding regions by database similarity search. Nature Genetics 1993, 3(3):266-272.

21. Altschul SF, Gish W, Miller W, Myers EW, Lipman DJ: Basic local alignment search tool. Journal of Molecular Biology 1990, 215(3):403-410 [http://www sciencedirect.com/science?_ob=MImg\&_imagekey=B6WK7-4N0J174-8$1 \& \_c d i=6899 \&$ _user=10\&_pii=S0022283605803602\&_origin=search\&_
zone=rslt_list_item\&_coverDate=10/05/1990\&_sk=997849996\&wchp= dGLzVzz-ZSkzS\&md5=010668abfa638d5457912865b7eaef37\&ie=/sdarticle. pdf].

22. Lartillot N, Philippe $\mathrm{H}$ : A Bayesian mixture model for across-site heterogeneities in the amino-acid replacement process. Molecular Biology and Evolution 2004, 21(6):1095-1109.

23. Huelsenbeck JP, Bull JJ: A likelihood ratio test to detect conflicting phylogenetic signal. Systematic Biology 1996, 45(1):92-98.

24. Legendre $P$, Desdevises $Y$, Bazin E: A statistical test for host-parasite coevolution. Systematic Biology 2002, 51(2):217-234.

25. Philippe H, Derelle R, Lopez P, Pick K, Borchiellini C, Boury-Esnault N, Vacelet J, Renard E, Houliston E, Queinnec E, et al: Phylogenomics revives traditional views on deep animal relationships. Current Biology 2009, 19(8):706-712.

26. Sperling EA, Peterson KJ, Pisani D: Phylogenetic-signal dissection of nuclear housekeeping genes supports the paraphyly of sponges and the monophyly of Eumetazoa. Molecular Biology and Evolution 2009, 26(10):2261-2274.

27. Shearer TL, Van Oppen MJH, Romano SL, Wörheide G: Slow mitochondrial DNA sequence evolution in the Anthozoa (Cnidaria). Molecular Ecology 2002, 11(12):2475-2487.

28. Swithers KS, Senejani AG, Fournier GP, Gogarten JP: Conservation of intron and intein insertion sites: implications for life histories of parasitic genetic elements. Bmc Evolutionary Biology 2009, 9.

29. Lynch $M$, Koskella B, Schaack S: Mutation pressure and the evolution of organelle genomic architecture. Science 2006, 311(5768):1727-1730.

30. Meroz-Fine E, Shefer S, llan M: Changes in morphology and physiology of an East Mediterranean sponge in different habitats. Marine Biology 2005, 147(1):243-250.

31. Chen $\mathrm{YH}$, Chen $\mathrm{CP}$, Chang $\mathrm{KH}$ : Budding cycle and bud morphology of the globe-shaped sponge Cinachyra australiensis. Zoological Studies 1997, 36(3):194-200.

32. Rützler K, Smith KP: Guide to Western Atlantic species of Cinachyrella (Porifera, Tetillidae). Proceedings of the Biological Society of Washington 1992, 105(1):148-164 [http://si-pddr.si.edu/dspace/bitstream/10088/7916/1/ iz_Ruetzler_Smith_1992.pdf].

33. Ereskovsky AV, Tokina DB: Asexual reproduction in homoscleromorph sponges (Porifera; Homoscleromorpha). Marine Biology 2007, 151(2):425-434.

34. Wörheide $G$, Erpenbeck D, Menke $C$ : The sponge barcoding project: aiding in the identification and description of poriferan taxa. In Porifera research: biodiversity, innovation and sustainability. Edited by: Custódio MR, Lôbo-Hajdu G, Hajdu E, Muricy G. Rio de Janeiro: Museu Nacional; 2007:28:123-128 [http://www.poriferabrasil.mn.ufrj.br/iss/].

35. Cárdenas P, Menegola C, Rapp HT, Diaz MC: Morphological description and DNA barcodes of shallow-water Tetractinellida (Porifera: Demospongiae) from Bocas del Toro, Panama, with description of a new species. Zootaxa 2009, , 2276: 1-39 [http://www.mapress.com/zootaxa/ 2009/f/Z02276p039f.pdf].

36. Gaino E, Liaci LS, Sciscioli M, Corriero G: Investigation of the budding process in Tethya citrina and Tethya aurantium (Porifera, Demospongiae). Zoomorphology 2006, 125(2):87-97.

37. Gaino E, Mercurio M, Sciscioli M, Corriero G: Choanocyte chambers in unreleased buds of Tethya seychellensis (Wright, 1881) (Porifera, Demospongiae). Italian Journal of Zoology 2009, 76(1):64-69.

38. Borchiellini C, Chombard C, Manuel M, Alivon E, Vacelet J, Boury-Esnault N: Molecular phylogeny of Demospongiae: implications for classification and scenarios of character evolution. Molecular Phylogenetics and Evolution 2004, 32(3):823-837.

39. Nichols SA: An evaluation of support for order-level monophyly and interrelationships within the class Demospongiae using partial data from the large subunit rDNA and cytochrome oxidase subunit I. Molecular Phylogenetics and Evolution 2005, 34(1):81-96.

40. McLeish MJ, Crespi BJ, Chapman TW, Schwarz MP: Parallel diversification of Australian gall-thrips on Acacia. Molecular Phylogenetics and Evolution 2007, 43(3):714-725.

41. Van Soest RWM, Hooper JNA: Family Tetillidae Solas, 1886. In Systema Porifera: A guide to the classification of sponges. Edited by: Hooper JNA, Van Soet EWM. New York: Kluwer Academic/Plenum Publishers; 2002:. 
42. Kim CS, Lee CH, Shin JS, Chung YS, Hyung NI: A simple and rapid method for isolation of high quality genomic DNA from fruit trees and conifers using PVP. Nucleic Acids Research 1997, 25(5):1085-1086.

43. Lisacek F, Diaz Y, Michel F: Automatic identification of group-I intron cores in genomic DNA-sequences. Journal of Molecular Biology 1994, 235(4):1206-1217.

44. Mathews DH, Sabina J, Zuker M, Turner DH: Expanded sequence dependence of thermodynamic parameters improves prediction of RNA secondary structure. Journal of Molecular Biology 1999, 288:911-940.

45. Zuker M: Mfold web server for nucleic acid folding and hybridization prediction. Nucleic Acids Research 2003, 31(13):3406-3415.

46. De Rijk P, De Wachter R: RnaViz, a program for the visualisation of RNA secondary structure. Nucleic Acids Research 1997, 25(22):4679-4684.

47. Katho K, Misawa K, Kuma K, Miyata T: MAFFT: a novel method for rapid multiple sequence alignment based on fast Fourier transform. Nucleic Acids Research 2002, 30(14):3059-3066.

48. Castresana J: Selection of conserved blocks from multiple alignments for their use in phylogenetic analysis. Molecular Biology and Evolution 2000, 17(4):540-552.

49. Loytynoja A, Milinkovitch MC: SOAP, cleaning multiple alignments from unstable blocks. Bioinformatics 2001, 17(6):573-574.

50. Stamatakis A: RAxML-VI-HPC: maximum likelihood-based phylogenetic analyses with thousands of taxa and mixed models. Bioinformatics 2006 22(21):2688-2690.

51. Lartillot N, Lepage T, Blanquart S: PhyloBayes 3: a Bayesian software package for phylogenetic reconstruction and molecular dating. Bioinformatics 2009, 25(17):2286-2288.

52. Suyama M, Torrents D, Bork P: PAL2NAL: robust conversion of protein sequence alignments into the corresponding codon alignments. Nucleic Acids Research 2006, 34:W609-W612.

53. Cho Y, Qiu YL, Kuhlman P, Palmer JD: Explosive invasion of plant mitochondria by a group I intron. Proceedings of the National Academy of Sciences of the USA 1998, 95(24):14244-14249.

54. Swofford DL: PAUP*. Phylogenetic analysis using parsimony (*and other methods). Sunderland, Massachusetts: Sinauer Associates, 4.

55. Posada D, Crandall KA: MODELTEST: testing the model of DNA substitution. Bioinformatics 1998, 14(9):817-818.

56. Shimodaira $\mathrm{H}$, Hasegawa M: CONSEL: for assessing the confidence of phylogenetic tree selection. Bioinformatics 2001, 17(12):1246-1247.

57. Maddison WP, Maddison DR: Mesquite: a modular system for evolutionary analysis. Version 2.72. 2009 [http://mesquiteproject.org]

58. Legendre P, Anderson MJ: Distance-based redundancy analysis: Testing multispecies responses in multifactorial ecological experiments. Ecological Monographs 1999, 69(4):512-512 [http:/www.esajournals.org/doi/ abs/10.1890/0012-9615\%281999\%29069\%5B0001:DBRATM\%5D2.0.CO;2].

59. Guindon S, Gascuel O: A simple, fast, and accurate algorithm to estimate large phylogenies by maximum likelihood. Syst Biol 2003, 52(5):696-704.

doi:10.1186/1471-2148-10-288

Cite this article as: Szitenberg et al:: Diversity of sponge mitochondrial introns revealed by cox 1 sequences of Tetillidae. BMC Evolutionary Biology 2010 10:288.

\section{Submit your next manuscript to BioMed Central and take full advantage of:}

- Convenient online submission

- Thorough peer review

- No space constraints or color figure charges

- Immediate publication on acceptance

- Inclusion in PubMed, CAS, Scopus and Google Scholar

- Research which is freely available for redistribution

Submit your manuscript at www.biomedcentral.com/submit 